\title{
Role of Diffusion Tensor Imaging Combined with Neuron-Specific Enolase and S100 Calcium-binding Protein B Detection in Predicting the Prognosis of Moderate and Severe Traumatic Brain Injury
}

\author{
Chen Peng ${ }^{1}$, Yu Xing ${ }^{1}$, Huang Tao ${ }^{1,}$, Deng Yongbing ${ }^{1}$ and Huang Jingrui ${ }^{1}$ \\ ${ }^{1}$ Department of Neurosurgery, Chongqing Emergency Medical Center, Chongqing University Central Hospital, Chongqing, China \\ * Corresponding author: Huang Tao, Huang Jingrui, Department of Neurosurgery, Chongqing Emergency Medical Center, Chongqing \\ University Central Hospital, Chongqing, China. Tel: 18680875255; Email: sunny.hjr@163.com
}

Received 2021 January 06; Revised 2021 January 26; Accepted 2021 March 13.

\begin{abstract}
Background: Traumatic brain injury (TBI) seriously affects the quality of life of patients. The present study evaluated the role of diffusion tensor imaging (DTI) combined with Neuron-Specific Enolase (NSE) and S100 calcium-binding protein B (S100B) protein in predicting the prognosis of moderate and severe TBI.

Methods: The TBI patients were divided into moderate TBI (TBIm) and severe TBI (TBIs) groups according to the Glasgow Coma Scale (GCS) after admission. The patients were then divided into good and poor prognosis groups according to the Glasgow Outcome Scale (GOS); moreover, their follow-ups were recorded at 3 and 6 months after injury. This study also included 65 healthy individuals with matched age and gender as the control group. The fractional anisotropy (FA) values of DTI, serum neuron-specific enolase (NSE), and S100B protein levels were detected in this study. The data were analyzed in SPSS software (version 22.0) to evaluate the role of DTI combined with NSE and S100B protein in predicting the prognosis in TBIm and TBIs.

Results: After TBI, the FA values of DTI in the TBI group were lower than those in the control group (P<0.05); moreover, the serum NSE and S100B values in the TBI group were higher than those in the control group $(\mathrm{P}<0.05)$. In the TBIm patients, the FA values of the corpus callosum in the good prognosis group were higher than that in the poor prognosis group $(\mathrm{P}<0.05)$; however, there was no significant difference between the two groups regarding the FA values of the internal capsule and the cerebral peduncle (P>0.05). The serum levels of NSE and S100B in the good prognosis group were significantly lower than those in the poor prognosis group (P<0.05). In the TBIs patients, the FA value of all areas in the good prognosis group was significantly higher than that in the poor prognosis group $(\mathrm{P}<0.05)$. However, there was no significant difference between the two prognosis groups regarding the serum levels of NSE and $\mathrm{S} 100 \mathrm{~B}(\mathrm{P}>0.05)$.

Conclusion: Although DTI combined with NSE and S100B protein can effectively predict the prognosis of patients with moderate and severe TBI in the early stages, various other measures have been used in the studies to predict the prognosis of TBI patients. Accordingly, comparison with other measures is essential in further studies.
\end{abstract}

Keywords: Diffusion tensor imaging, Neuron-specific enolase, Prognosis evaluation, S100B Protein, Traumatic brain injury

\section{Background}

Traumatic brain injury (TBI) is a common form of trauma, the incidence of which increases year by year $(1,2)$. Acute TBI and its complications seriously affect the quality of life of patients and even directly threaten the lives of patients, bringing a heavy burden to their families and society. At present, in patients with acute TBI, condition evaluation and prognosis prediction mainly rely on the Glasgow Coma Scale (GCS) score and brain computed tomography (CT) scan imaging data (3, 4). However, there was no intensive research on the prediction of prognosis of moderate TBI (TBIm) or severe TBI (TBIs) in the early stage. In addition, most of such prediction research was based on single-factor analysis; moreover, they lack comprehensive multi-factor prediction and assessment.

Diffusion tensor imaging (DTI) is a magnetic resonance imaging method by establishing images of the anisotropy of diffuse motion of water molecules. With indicators, such as fractional anisotropy (FA) and average diffusion coefficient provided by DTI, it can indirectly reflect the structural integrity of nerve bundle; accordingly, DTI has an exact effect on the diagnosis of brain stem injury and diffuse axonal injury $(5,6)$. Researchers found that in brain injury, FA value by DTI was inversely proportional to the size of lesions in TBI, that is to say, patients with higher FA value have a better prognosis $(7,8)$.

However, whether the FA value in patients with TBIm or TBIs has a linear inverse relationship with their prognosis is unclear; moreover, only relying on DTI cannot provide a comprehensive assessment for the TBI patients. In recent years, studies have found that the detection of some nervous system-related proteins in the blood played a significant role in evaluating the degree of TBI and determining the prognosis (9), including neuron-specific enolase (NSE) and S100B protein. 
The NSE participates in the metabolic process of glycolysis, and its release is increased when the integrity of the neuron membrane is compromised (10). S100B protein exists mainly in astrocytes and Schwann cells. When the cells of the central nervous system are damaged after TBI, S100B protein will escape from the cerebrospinal fluid to the blood through the damaged blood-brain barrier (11). Therefore, it is concluded that both NSE and S100B protein contents in serum can reflect the degree and range of brain injury and serve as biochemical markers to estimate injury condition and prognosis (12).

In this study, DTI combined with serum NSE and S100B protein was applied to evaluate the prognosis of patients with TBIm or TBIs. Moreover, it attempted to explore the value of imaging combined with changes in biochemical indicators to predict the prognosis of TBIm or TBIs and provide a theoretical basis for early estimation of prognosis and prompt adjustment therapy after TBI.

\section{Methods}

\subsection{Clinical data}

This retrospective study investigated the clinical data of 74 patients ( 40 males and 34 females) with TBIm or TBIs who were admitted to the Department of Neurosurgery of Chongqing Emergency Medical Center from March 2016 to January 2019. The mean age of the patients was obtained at $34.2 \pm 8.2$ years. All the patients had clear injury causes, including traffic accidents $(\mathrm{n}=34)$, high falling accidents $(n=20)$, falling injuries $(n=12)$, and beaten injuries $(\mathrm{n}=8)$.

Furthermore, 65 healthy cases of the same age (mean age: $36.1 \pm 7.9$ years) and gender ( 35 males and 30 females) were included in this study as the control group. It should be mentioned that those with a history of nervous and mental diseases, brain injury, and surgery were excluded from the study. Moreover, there was no significant difference between the control and experimental groups regarding gender and age.

The inclusion criteria were: 1) a clear history of TBI, 2) admission within $24 \mathrm{~h}$ after injury, 3) age of 16-60 years, 4) confirmation of TBI by brain CT scan, and 5) GCS score of 6-12. On the other hand, those with severe associated injuries in other sites, such as thorax, abdomen, spine and limbs; previous history of neurological diseases, such as brain tumor, cerebral hemorrhage, brain abscess, cerebral infarction; a previous history of mental illness; severe heart, lung, liver, and kidney diseases, as well those who deceased within $24 \mathrm{~h}$ after admission were excluded from the study.

The study protocol was approved by the Ethics Committee of Chongqing Emergency Medical Center, Chongqing, China. Regarding the ethical consideration, all patients and/or their family members, and volunteers were informed of the research objectives and procedures. Moreover, informed consent was obtained from them.

\subsection{Grouping}

Injury assessment: The degree of injury of the TBI patients was rated after admission according to GCS. The GCS assessed eyes, as well as verbal and motor reactions. The total score was considered 15, and lower scores were associated with more serious conditions. Furthermore, the scores more than 13, 9-12, and 3-8 suggested slight, injury, and severe injuries, respectively. It is worth mentioning that the TBI patients with GCS score of 6-12 were included in this study, and they were then divided into two groups of moderate (GCS:9-12; $n=46$; regarded as TBIm) and severe (GCS: 3-8; $\mathrm{n}=28$; regarded as TBIs).

Prognosis assessment: The included cases were scored using Glasgow Outcome Scale (GOS) after 3 and 6 months post injury in a visit by a physician. According to the GOS score, the TBIm and TBIs groups by injury grouping were further divided into groups with good prognosis (GOS: 4-5) and poor prognosis (GOS: 1-3).

\subsection{Observational indicators}

\subsubsection{Diffusion tensor imaging (DTI)}

All patients' examinations were performed one week after admission. Furthermore, magnetic resonance imaging was carried out using GE Signa Excite HD 1.5t superconducting magnetic resonance scanner with 8-channel phased-array coils. The scanning range covered the whole brain. The conventional scanning sequence included T1WI, T2WI, T1WI, TR/TE $=400 \mathrm{~ms} / 15 \mathrm{~ms}, \quad \mathrm{~T} 2 \mathrm{WI}$, and $\mathrm{TR} / \mathrm{TE}=3000 \mathrm{~ms} / 100 \mathrm{~ms}$; additionally, the layer thickness and spacing were obtained at 6 and $1 \mathrm{~mm}$ respectively. In diffusion tensor imaging (DTI), the values of diffusion sensitive coefficient were determined at $0 \mathrm{~s} / \mathrm{mm}^{2}$ and $1000 \mathrm{~s} / \mathrm{mm}^{2}$, and a gradient magnetic field was applied in 25 directions. The main parameters were TR $/ \mathrm{TE}=10000 \mathrm{~ms} / 95 \mathrm{~ms}$, matrix $128 \times 128$, and FOV $=24 \mathrm{~cm}$; moreover, layer thickness and spacing were determined at 4 and 0 $\mathrm{mm}$, respectively. The DTI data were transferred to the GE ADW 4.4 workstation, and the Functool software was applied for post-processing to obtain the FA diagram of each layer. The FA values in the corpus callosum, internal capsule, and cerebral peduncle were mainly measured in this study. In total, three to five ROIs were successively set from the genu of the corpus callosum to the splenium of the corpus callosum. In addition, the internal capsule and cerebral peduncle were measured on both sides, with 2 to 4 ROI on each side, and the ROI size was about 20 to 50 pixels (equivalent to 10 to $30 \mathrm{~mm}^{2}$ ). 


\subsubsection{Detection of NSE and $S 100 B$}

Blood samples were taken from the cubital vein of all patients $24 \mathrm{~h}$ after injury. After coagulation, the blood was centrifuged at $4^{\circ} \mathrm{C}$, and the serum was separated. NSE and S100Bprotein were determined by ELISA and the corresponding kit was selected. The NSE and the S100B kits were purchased from Wuhan Jiyimei Biotechnology Co., LTD. (JYM1105Hu) and Shanghai Beyotime Biotechnology Co., LTD. (PS842), respectively. The measurements were carried out strictly following the instructions.

\subsection{Statistical method}

The obtained data were analyzed in SPSS software (version 22.0). The categorical data were represented as rate (\%) and analyzed through the chi-square test. The variable data were consisted with normal distribution were represented as mean \pm standard deviation $(\mathrm{X} \pm \mathrm{SD})$. On the other hand, the variable data that did not conform to the normal distribution were represented by the median ( $\mathrm{M}$, range) and analyzed using the Kolmogorov-Smirnov test. A p-value less than 0.05 was considered statistically significant.

\section{Results}

\subsection{Results and comparison of DTI in different TBI} groups and prognosis groups (Table 1)

\subsubsection{Comparison of FA values in different TBI groups}

As can be observed in Table 1, after one week post injury, the FA values at each interesting region of TBIm and TBIs are significantly lower than those of the control group $(\mathrm{P}<0.05)$. Moreover, the FA values at each interesting region of the TBIs group are lower than those of the TBIm group $(\mathrm{P}<0.05)$.
3.1.2. Comparison of FA values in different prognostic groups in patients with TBIm

According to the results, the FA value in the corpus callosum of the good prognosis group was higher than that of the poor prognosis group $(\mathrm{P}<0.05)$; however, there was no difference between the regions of the internal capsule and cerebral peduncle in different prognosis groups regarding the FA values $(\mathrm{P}>0.05)$ (Table 2).

\subsubsection{Comparison of FA values in different prognostic} groups in patients with TBIs

It was also found that the FA value of the good prognosis group was significantly higher than that of the poor prognosis group at each interesting region $(\mathrm{P}<0.05)$ (Table 2).

3.2. Results and comparison of serum biochemical indexes (NSE and S100B) in different TBI groups and prognosis groups

3.2.1. Comparison of serum levels of NSE and S100B in different TBI groups.

The results of Table 3 indicate that $24 \mathrm{~h}$ after injury, the serum levels of NSE and S100B in TBIm and TBIs are higher than those of the control group $(\mathrm{P}<0.05)$. However, serum levels of NSE and S100B in the TBIs group are not statistically different from those in the TBIm group.

\subsubsection{Comparison of serum levels of NSE and S100B in} different prognostic groups in patients with TBIm

The results showed that the serum levels of both NSE and S100B in the good prognosis group were significantly lower than those in the poor prognosis group $(\mathrm{P}<0.05)$ (Table 4$)$.

\begin{tabular}{lcccccc}
\hline \multicolumn{2}{l}{ Table 1. Comparison of FA values at each interesting region in different TBI groups } & & & \\
\hline Region & control $(\mathbf{n}=\mathbf{6 5})$ & TBIm(n=46) & TBIs(n=28) & P* & $\mathbf{P}^{* *}$ & P*** \\
\hline Corpus callosum & $0.71 \pm 0.08$ & $0.63 \pm 0.05$ & $0.55 \pm 0.05$ & 0.109 & 0.001 & 0.001 \\
\hline Internal capsule & $0.59 \pm 0.11$ & $0.53 \pm 0.05$ & $0.47 \pm 0.03$ & 0.058 & 0.001 & 0.001 \\
\hline Cerebral peduncle & $0.63 \pm 0.08$ & $0.57 \pm 0.04$ & $0.48 \pm 0.06$ & 0.094 & 0.001 & 0.001 \\
\hline
\end{tabular}

*Control group, compared to TBIm

** Control group, compared to the TBIs group

***TBIs group, compared to the TBIm group

\begin{tabular}{|c|c|c|c|c|c|c|}
\hline \multirow{2}{*}{ Region } & \multicolumn{2}{|c|}{ TBIm(n=46) } & \multirow{2}{*}{$\mathbf{P}$} & \multicolumn{2}{|c|}{ TBIs(n=28) } & \multirow{2}{*}{$\mathbf{P}$} \\
\hline & Good prognosis & Poor prognosis & & Good prognosis & Poor prognosis & \\
\hline Corpus callosum & $0.65 \pm 0.07$ & $0.61 \pm 0.04$ & 0.031 & $0.57 \pm 0.06$ & $0.52 \pm 0.03$ & 0.001 \\
\hline Internal capsule & $0.54 \pm 0.06$ & $0.53 \pm 0.07$ & 0.653 & $0.50 \pm 0.05$ & $0.45 \pm 0.02$ & 0.001 \\
\hline Cerebral peduncle & $0.56 \pm 0.08$ & $0.57 \pm 0.06$ & 0.317 & $0.52 \pm 0.04$ & $0.44 \pm 0.04$ & 0.001 \\
\hline
\end{tabular}

\begin{tabular}{|c|c|c|c|c|c|}
\hline Indicator & $\begin{array}{c}\text { Control } \\
(n=65)\end{array}$ & $\begin{array}{c}\text { TBIm } \\
(n=46)\end{array}$ & $\mathbf{P}^{*}$ & $\begin{array}{c}\text { TBIs } \\
(n=28)\end{array}$ & $\mathbf{P}^{*}$ \\
\hline NSE (ng/ml) & $10.12 \pm 1.78$ & $37.93 \pm 5.85$ & 0.001 & $39.55 \pm 8.15$ & 0.001 \\
\hline S100B (ug/L) & $0.19 \pm 0.03$ & $0.68 \pm 0.14$ & 0.001 & $0.83 \pm 0.16$ & 0.001 \\
\hline
\end{tabular}

*Compared to control 
Table 4. Comparison of serum levels of NSE and S100B in different prognostic groups with TBIm and TBIs

\begin{tabular}{|c|c|c|c|c|c|c|}
\hline \multirow{2}{*}{ Indicator } & \multicolumn{2}{|c|}{$\operatorname{TBIm}(n=46)$} & \multirow{2}{*}{$\mathbf{P}$} & \multicolumn{2}{|c|}{ TBIs(n=28) } & \multirow{2}{*}{$\mathbf{P}$} \\
\hline & Good prognosis & Poor prognosis & & Good prognosis & Poor prognosis & \\
\hline NSE (ng/ml) & $31.09 \pm 5.68$ & $47.78 \pm 9.43$ & 0.001 & $38.29 \pm 6.08$ & $42.26 \pm 8.43$ & 0.056 \\
\hline S100B (ug/L) & $0.55 \pm 0.08$ & $0.74 \pm 0.11$ & 0.001 & $0.80 \pm 0.12$ & $0.93 \pm 0.21$ & 0.179 \\
\hline
\end{tabular}

\subsubsection{Comparison of serum levels of NSE and S100B in} different prognostic groups in patients with TBIs

It was further observed that the serum levels of NSE and S100B were not statistically different in various prognosis groups (Table4).

\section{Discussion}

Acute TBI is an important cause of death and disability in young adults (2); accordingly, it is important to receive timely and effective treatment. For moderate and severe TBI, if the prognosis can be predicted early after injury, it is significant for recovery and timely adjustment therapy. At present, the early prognosis of TBI is mainly determined by GCS and traditional imaging examination, such as CT (4); however, both have limitations of inaccuracy. The researchers have found that GCS and traditional imaging manifestations were not strongly correlated with prognosis, and patients with diffuse axonal injury have no obvious imaging manifestations but their clinical conditions are severe and their prognosis is poor $(13,14)$.

The cerebral cortex and white matter may be damaged after TBI. For white matter damage, magnetic resonance DTI can not only display the site of damage visually but also quantify the lesion degree and analysis damage type using a series of data, such as FA value. In case of moderate and severe TBI, neuraxons are damaged, axoplasmic transport is obstructed, and water molecules diffusion in the direction parallel to the axons are restricted, resulting in a decrease in FA value in the acute phase (15). Other studies have found that the FA value in the TBI patients with poor prognosis is more significantly lower than that in patients with good prognosis (16), indicating that patients with a mild injury can maintain better axonal integrity, resulting in the better recovery and regeneration, compared to those with severe injury. While few previous studies have assessed DTI indices along with biochemical proteins, such as SE and S100B, the present study evaluated the associations of these factors. Regarding the cortex damage, it has been shown that the concentration of NSE and S100B protein in serum is closely related to the degree of primary cortex damage (17). Consistent with the results of the current study, Mercier et al. showed that the levels of NSE and S100B had a positive correlation with TBI, indicating that these indicators could be increased with the aggravation of TBI (18). The present study combined the early results of DTI imaging and serum NSE, as well as S100B protein biochemical markers in patients with moderate and severe TBI, and observed their relationship with patients' prognosis. Furthermore, it showed the value of DTI combined with NSE and S100B in the early evaluation of the prognosis of patients with moderate and severe TBI.

\subsection{Reltionship between combined results and prognosis in patients with moderate TBI}

Patients with TBIm were divided into good and poor prognosis groups according to GOS. Considering the relationship between the FA value of DTI imaging and the prognosis, the results showed that only in the region of the corpus callosum, the FA value of the good prognosis group was higher than that of the poor prognosis group. However, there was no difference in the FA values in the regions of the internal capsule and cerebral peduncle in different prognosis groups. It was also indicated that for TBIm patients, the FA value of early DTI examination was less sensitive to predict prognosis. As for the relationship among the early detection of NSE, S100B, and prognosis, it was found that the serum levels of both NSE and S100B in the good prognosis group were significantly lower than those in the poor prognosis group, suggesting that in the TBIm patients, an increase in serum levels of early NSE and S100B could directly reflect the prognosis, and the sensitivity was also superior to DTI.

\subsection{Relationship between combined results and prognosis in patients with severe TBI}

The patients with TBIs were also divided into good and poor prognosis groups. Considering the relationship between DTI results and prognosis, it was found that in the regions of the corpus callosum, internal capsule, and cerebral peduncle, the FA value of the good prognosis group was significantly higher than that of poor prognosis group. This indicated that the FA value of early DTI examination in TBIs patients was highly sensitive to predict prognosis, which was significantly different from the results obtained from the TBIm patients. As for the relationship among the early detection of NSE, S100B, and prognosis, the results showed no significant difference between the two prognosis groups regarding the serum NSE and S100B levels. This also suggested that the serum level of early NSE and S100B in the TBIs patients could not effectively predict the prognosis, and the sensitivity was poor, which was significantly different from the results obtained from the TBIm patients.

Park et al. showed that the level of serum S100B 
one week after TBI was correlated with the severity of brain injury, which was consistent with the results of our study. These findings suggested that in pediatric TBI patients, serum S100B and NSE might play a role in predicting the prognosis and tracking continuing brain injury (19). However, a significant relationship was observed between both biomarkers and brain damage along with prognosis of the traumatic injury in this study. Thelin et al. only found NSE to be a predictor of prognosis of the traumatic brain injury (20).

One of the limitations of this study was the wide interval between two follow-ups ( 3 and 6 months) for the prognostic evaluation.

\section{Conclusion}

In conclusion, DTI imaging combined with serum NSE and S100B levels can effectively predict the prognosis of patients with moderate and severe TBI in the early stage. Moreover, there are differences in TBIm and TBIs patients. For the prognosis of TBIm patients, serum biochemical indices are more direct and effective, while the result of DTI is significant only in the region of corpus callosum. For the prognosis of TBIs patients, the results of DTI in each region can be effective for determination, while the serum biochemical indicators are not significantly effective.

\section{Acknowledgments}

The authors express their gratitude to the staff of the Neurosurgery Intensive Care Unit of Chongqing Emergency Medical Center, Chongqing, China, who have made this study possible.

\section{Footnotes}

Authors' Contribution: Huang Jingrui and Chen Peng participated in the design and coordination of the study and drafted the manuscript. Chen Peng, Yu Xing, and Deng Yongbing participated in the clinical evaluation of the patients. Deng Yongbing and Huang Tao interpreted the imaging studies. Chen Peng and Yu Xing performed the statistical analysis. All authors read and approved the final manuscript.

Conflict of Interests: The authors have no conflict of interest to declare.

Ethical Approval: This study was approved by the Ethics Committee of Chongqing Emergency Medical Center, Chongqing, China (2017-8).

Funding/Support: This study was financially supported by the Medical Research Centre of Health and Family Planning Commission of Chongqing, China (2017ZDXM024).

Financial Disclosure: None declared.

Informed consent: Informed consent was obtained from all individual participants included in the study.

\section{References}

1. Galgano M, Toshkezi G, Qiu X, Russell T, Chin L, Zhao LR Traumatic brain injury: current treatment strategies and future endeavors. Cell Transplant. 2017;26(7):1118-30. doi: 10.1177/0963689717714102. [PubMed: 28933211].

2. Nguyen R, Fiest KM, McChesney J, Kwon CS, Jette N, Frolkis AD, et al. The international incidence of traumatic brain injury: a systematic review and meta-analysis. Can J Neurol Sci. 2016;43(6):774-85. doi: 10.1017/cjn.2016.290. [PubMed: 27670907].

3. Duncan CC, Summers AC, Perla EJ, Coburn KL, Mirsky AF. Evaluation of traumatic brain injury: brain potentials in diagnosis, function, and prognosis. Int J Psychophysiol. 2011; 82(1):24-40. doi: 10.1016/j.ijpsycho.2011.02.013. [PubMed: 21356253].

4. Guo H, Liu R, Sun Z, Liu B, Xiang Y, Mao J, et al. Evaluation of prognosis in patients with severe traumatic brain injury using resting-state functional magnetic resonance imaging. World Neurosurg. 2019;121:e630-9. doi: 10.1016/j.wneu.2018.09.178. [PubMed: 30292041].

5. Kraus MF, Susmaras T, Caughlin BP, Walker CJ, Sweeney JA, Little DM. White matter integrity and cognition in chronic traumatic brain injury: a diffusion tensor imaging study. Brain. 2007; 130(Pt 10):2508-19. doi: 10.1093/brain/awm216. [PubMed: 17872928].

6. Main KL, Soman S, Pestilli F, Furst A, Noda A, Hernandez B, et al. DTI measures identify mild and moderate TBI cases among patients with complex health problems: a receiver operating characteristic analysis of U.S. veterans. Neuroimage Clin. 2017;16:1-16. doi: 10.1016/j.nicl.2017.06.031. [PubMed: 28725550].

7. Edlow BL, Copen WA, Izzy S, Bakhadirov K, van der Kouwe A, Glenn MB, et al. Diffusion tensor imaging in acute-to-subacute traumatic brain injury: a longitudinal analysis. BMC Neurol. 2016;16:2. doi: 10.1186/s12883-015-0525-8. [PubMed: 26754948].

8. Wallace EJ, Mathias JL, Ward L. Diffusion tensor imaging changes following mild, moderate and severe adult traumatic brain injury: a meta-analysis. Brain Imaging Behav. 2018;12(6):16071621. doi: 10.1007/s11682-018-9823-2. [PubMed: 29383621].

9. Strathmann FG, Schulte S, Goerl K, Petron DJ. Blood-based biomarkers for traumatic brain injury: evaluation of research approaches, available methods and potential utility from the clinician and clinical laboratory perspectives. Clin Biochem. 2014;47(10-11):876-88.

doi: 10.1016/j.clinbiochem.2014.01.028. [PubMed: 24486649].

10. Cheng F, Yuan Q, Yang J, Wang W, Liu H. The prognostic value of serum neuron-specific enolase in traumatic brain injury: systematic review and meta-analysis. PLoS One. 2014; 9(9):e106680. doi: 10.1371/journal.pone.0106680. [PubMed: 25188406].

11. Vogt N, Herden C, Roeb E, Roderfeld M, Eschbach D, Steinfeldt $\mathrm{T}$, et al. Cerebral alterations following experimental multiple trauma and hemorrhagic shock. Shock. 2018;49(2):164-73. doi: 10.1097/SHK.0000000000000943. [PubMed: 28682946].

12. Ondruschka B, Pohlers D, Sommer G, Schober K, Teupser D, Franke $\mathrm{H}$, et al. S100B and NSE as useful postmortem biochemical markers of traumatic brain injury in autopsy cases. J Neurotrauma. 2013;30(22):1862-71. doi: 10.1089/neu.2013.2895. [PubMed: 23796187].

13. Currie S, Saleem N, Straiton JA, Macmullen-Price J, Warren DJ, Craven IJ. Imaging assessment of traumatic brain injury. Postgrad Med J. 2016;92(1083):41-50. doi: 10.1136/postgradmedj-2014133211. [PubMed: 26621823].

14. Mutch CA, Talbott JF, Gean A. Imaging evaluation of acute traumatic brain injury. Neurosurg Clin N Am. 2016;27(4):40939. doi: 10.1016/j.nec.2016.05.011. [PubMed: 27637393].

15. Maller JJ, Thomson RH, Lewis PM, Rose SE, Pannek K, Fitzgerald PB. Traumatic brain injury, major depression, and diffusion tensor imaging: making connections. Brain Res Rev. 2010;64(1):213-40. doi: 10.1016/j.brainresrev.2010.04.003. [PubMed: 20388528]. 
16. Roberts RM, Mathias JL, Rose SE. Diffusion Tensor Imaging (DTI) findings following pediatric non-penetrating TBI: a meta-analysis. Dev Neuropsychol. 2014;39(8):600-37. doi: 10.1080/87565641.2014.973958. [PubMed: 25470224].

17. Shinozaki K, Oda S, Sadahiro T, Nakamura M, Hirayama Y, Abe $\mathrm{R}$, et al. S-100B and neuron-specific enolase as predictors of neurological outcome in patients after cardiac arrest and return of spontaneous circulation: a systematic review. Crit Care. 2009;13(4):R121. doi: 10.1186/cc7973. [PubMed: 19624826].

18. Mercier E, Tardif PA, Cameron PA, Émond M, Moore L, Mitra B, et al. Prognostic value of neuron-specific enolase (NSE) for prediction of post-concussion symptoms following a mild traumatic brain injury: a systematic review. Brain Inj. 2018;32(1):29-40. doi: 10.1080/02699052.2017.1385097. [PubMed: 29157007].

19. Park DW, Park SH, Hwang SK. Serial measurement of S100B and NSE in pediatric traumatic brain injury. Childs Nerv Syst. 2019;35(2):343-8. doi: 10.1007/s00381-018-3955-y. [PubMed: 30171330].

20. Thelin EP, Jeppsson E, Frostell A, Svensson M, Mondello S, Bellander BM, et al. Utility of neuron-specific enolase in traumatic brain injury; relations to S100B levels, outcome, and extracranial injury severity. Crit Care. 2016;20:285. doi: 10.1186/s13054-016-1450-y. [PubMed: 27604350] 